\title{
A Japanese single-center experience of the efficacy and safety of asfotase alfa in pediatric-onset hypophosphatasia
}

\author{
Yohei Sugiyama ${ }^{1,2,3 \dagger}$, Taijiro Watanabe ${ }^{2 \dagger}$, Makiko Tajika ${ }^{1,2 \dagger}$, Tetsuro Matsuhashi ${ }^{1,2}$, Masaru Shimura ${ }^{1,2}$, \\ Takuya Fushimi 1,2, Keiko Ichimoto ${ }^{1,2}$, Ayako Matsunaga ${ }^{1,2}$ (D) Tomohiro Ebihara ${ }^{1,3}$, Tomoko Tsuruoka ${ }^{1,3}$, \\ Tomoyuki Akiyama ${ }^{4}$ and Kei Murayama ${ }^{1,2^{*}}$ (1)
}

\begin{abstract}
Background: Hypophosphatasia (HPP) is a rare inherited metabolic disorder caused by mutations in the ALPL gene, which encodes tissue nonspecific alkaline phosphatase. The severity of HPP is widely diverse from the perinatal form to the adult mild form. The former represents the most severe form and was earlier associated with high mortality due to pneumonia which was caused by severe hypomineralization of the bones-such as chest deformity and fractured ribs - and muscle weakness. Enzyme replacement therapy using asfotase alfa (AA) was approved in 2015 in Japan for treating patients with HPP and has improved their pulmonary function and life prognosis. There are several practical and ethical challenges related to using orphan drugs for a rare disorder in a publicly funded healthcare system. Sharing experiences about their application is essential towards formulating guidelines to assist clinicians with decisions about their initiation and withdrawal. We report the details of AA experience in ten cases of pediatric-onset HPP in nine families from January 2015 to November 2019 (median [interquartile range] age 11.0 [7.6-12.5] years; 60\% male). This is a study of a single-center cohort describing the clinical course of patients with HPP, mainly consisting of the mild childhood form of HPP, treated with AA in Japan.
\end{abstract}

Results: One case of perinatal form of HPP, two cases of benign prenatal form, and seven cases of childhood form were observed. The most common symptom at onset was pain. All patients had low serum alkaline phosphatase levels as compared to the age-matched reference range before the commencement of AA. All HPP patients seem to have responded to AA treatment, as evidenced by pain alleviation, increased height standard deviation, improvement in respiratory condition and 6-min walk test result improvement, disappearance of kidney calcification, alleviation of fatigue, and/or increases in bone mineralization. There were no serious adverse events, but all patients had an injection site reaction and skin changes at the injection sites. Genetic analysis showed that eight out of ten patients had compound heterozygosity.

Conclusions: AA may be effective in patients with mild to severe pediatric-onset forms of HPP.

Keywords: Alkaline phosphatase, Asfotase alfa, Enzyme replacement therapy, Hypophosphatasia

*Correspondence: kmuraya@mri.biglobe.ne.jp

${ }^{\dagger}$ Yohei Sugiyama, Taijiro Watanabe and Makiko Tajika have contributed equally to this work

${ }^{1}$ Center for Medical Genetics, Chiba Children's Hospital, 579-1 Heta-cho, Midori-ku, Chiba City, Chiba Prefecture 266-0007, Japan

Full list of author information is available at the end of the article

\section{Background}

Hypophosphatasia (HPP) is a genetic disorder caused by defects in tissue-nonspecific alkaline phosphatase (TNSALP) [1]. The loss-of-function mutations in the $A L P L$ gene, located in the short arm of chromosome 1 , 
consisting of 12 exons, and encoding TNSALP, results in HPP [2]. ALPL variants occur in all exons. Depletion of TNSALP causes accumulation of phosphoethanolamine (PEA), pyridoxal 5' phosphate (PLP), and inorganic pyrophosphate. TNSALP dephosphorylates PLP into pyridoxal (PL), which can cross the blood-brain barrier and subsequently become rephosphorylated to PLP. In the brain, PLP is required for the biosynthesis of many neurotransmitters [3]. The central pathogenesis of HPP is impaired bone calcification, and the mechanism by which reduced TNSALP activity leads to hypocalcification is well understood [4]. TNSALP produces inorganic phosphate by degrading pyrophosphate, a calcification inhibitor [5]. The inorganic phosphate produced is taken up into the matrix vesicles released from osteoblasts $[6$, 7]. In HPP, it is thought that the accumulation of pyrophosphate and the decrease in local phosphorus concentration due to the decreased activity of TNSALP causes hypocalcification [8].

Patients with HPP have varied clinical manifestations and are classified as having one of the six forms (perinatal, benign prenatal, infantile, childhood, adult, and odontohypophosphatasia) based on the age of onset and severity $[1,9,10]$. There is a Japanese clinical practice guideline to diagnose patients with pediatric and adult HPP [11]. HPP has diverse symptoms, such as seizures, poor body weight gain, and premature deciduous tooth loss due to decrement in serum alkaline phosphate (ALP) levels [12]. A low ALP level is important for the diagnosis of HPP. HPP is diagnosed based on clinical symptoms, radiological findings, and biochemical test results by clinicians who are familiar with the disease. Genetic testing is recommended for supporting diagnosis and genetic counseling $[11,13]$. The incidence of severe phenotypes in Japan is approximately 1 in 150,000, whereas the incidence of other phenotypes is unclear [11]. In Japan, the common ALPL variants are c.1559delT and c.979T $>$ C. The prevalence of the c.1559delT allele is approximately $1 / 480$ [10]. Genetic carriers of HPP are usually asymptomatic, although some are mildly symptomatic. It is observed that the more severe the disease, the more often it is subject to autosomal recessive inheritance [14].

Enzyme replacement therapy using asfotase alfa (AA) has been approved in many countries, including the United States, the European Union, Canada, and Japan. A Japanese clinical trial has demonstrated the efficacy of AA in treating patients with HPP [15]. There were 13 patients in this trial: six with perinatal form, five with infantile form, one with childhood form, and one with the adult form of HPP. In Japan, AA is covered by health insurance, and the guidelines for its use as an established treatment protocol in patients with HPP facilitate its accessibility and availability to healthcare professionals and patients living with HPP as compared with these in other countries that do not provide insurance coverage or do not have regulatory approval for this treatment for adult-onset HPP [11]. There is some literature describing improvements in the clinical course of the infantile and childhood form of HPP, with rachitic change after AA treatment [16]. Nevertheless, it is necessary to share experiences from various centers to generalize the applicability of this novel pharmacotherapy for HPP; thus, we present a case series of pediatric-onset HPP and report our experience in treating patients with HPP, particularly the mild childhood form, with AA at our center.

\section{Methods}

This is the observational retrospective patient study and did not involve any research-based patient intervention. All Japanese patients with HPP who visited Chiba Children's Hospital, Japan between January 2015 and November 2019 were included in this study. The diagnosis of HPP was established based on the criteria laid down by the Japanese Clinical Practice Guidelines for HPP [11]. The guidelines define the primary clinical symptoms as bone mineralization disorder and premature loss of deciduous teeth, and the primary laboratory finding as low serum ALP levels compared with age-specific normal values [17]. If one or more primary symptoms are observed on clinical examination along with a low serum ALP level, HPP should be suspected, and gene testing would be performed for supporting diagnosis. This Japanese guideline is for diagnosis and states the recommendations of AA indication.

$A L P L$ gene analysis was performed with informed consent. Ten children ( 4 girls and 6 boys) from 9 families were included. The demographic characteristics, clinical features, laboratory investigations, and genetic analysis of all patients are summarized in Table 1. The classification of the clinical form of HPP was based on age at onset, symptoms, and severity of radiological findings [18]. The serum PLP, PL, and PLP/PL level reference values were determined based on a study published by Akiyama et al. [19], and that for PEA was from LSI Medicine Corporation. The serum ALP level reference values were determined based on a study published by Tanaka et al. [17]. Short stature was defined as a height of -2 standard deviation (SD) below the mean for the respective age as specified by the 2000 growth survey on body mass index for age references for Japanese children (crosssectional national survey data by the Japanese Ministry of Health, Labour and Welfare, and the Ministry of Education, Culture, Sports, Science, and Technology) [20, 21]. AA treatment involved subcutaneous administration thrice weekly at a dose of $2 \mathrm{mg} / \mathrm{kg}$ or six times a week at a dose of $1 \mathrm{mg} / \mathrm{kg}$, with the maximum volume of a single 


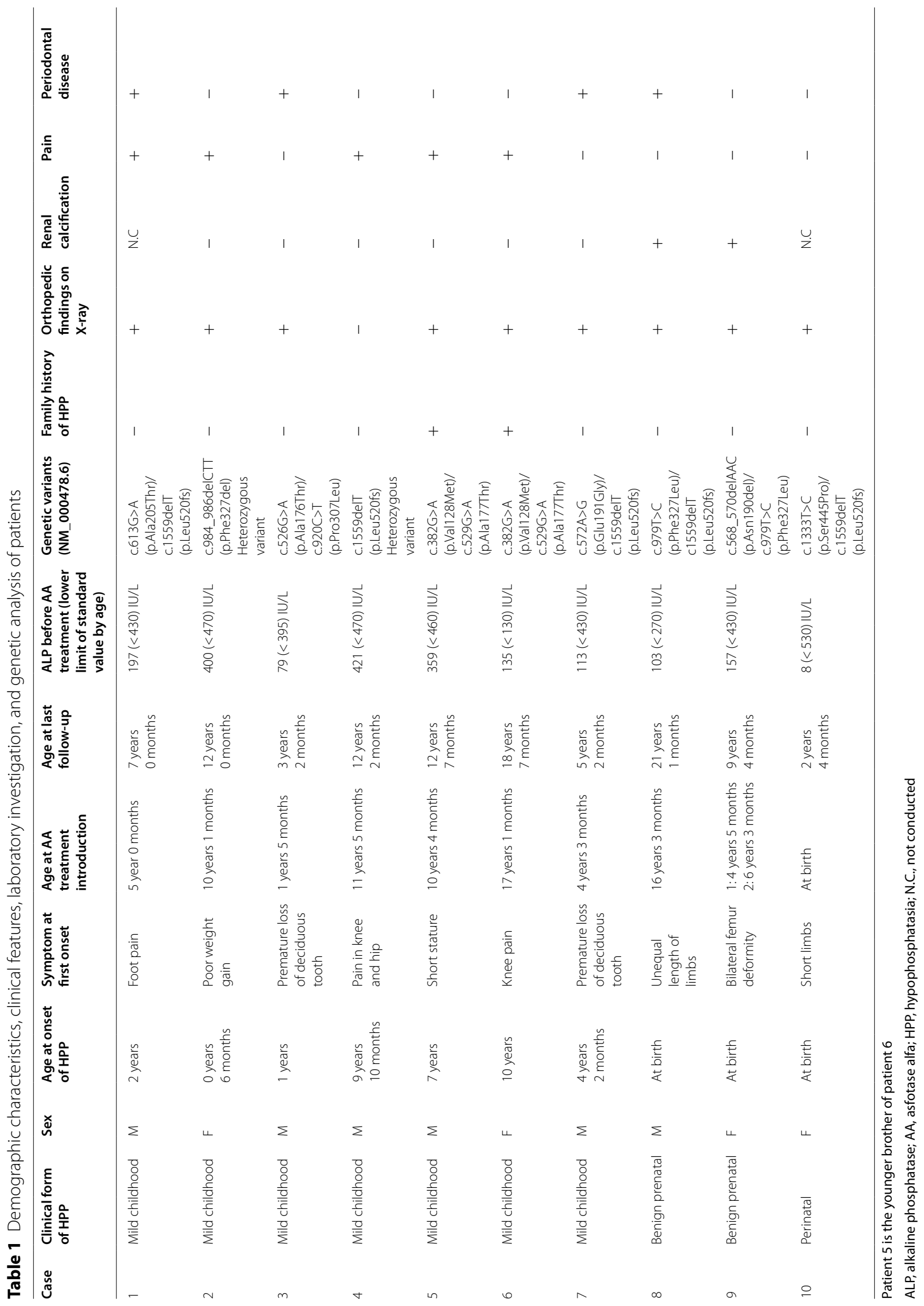


injection being $1 \mathrm{~mL}$ ( 40 or $100 \mathrm{mg} / \mathrm{mL}$ ). We routinely measured the bone density using dual X-ray absorption (DXA), biochemical data, kidney echography and carpus radiography data, limb pain, and 6-min walk test (6MWT) results depending on the symptoms and development of patients.

All diagnostic procedures and treatment protocols were based on established protocols intended to diagnose and treat patients, and adhered to the principles of the Declaration of Helsinki 1975, as revised in 2000. The assay for pyridoxal phosphate and pyridoxal was approved by the Research Ethics Board at Okayama University Hospital (Approval No. 1603-012).

\section{Results}

\section{Clinical data of patients with HPP}

The cases discussed here consist of one case of perinatal form, two cases of benign prenatal form, and seven cases of mild childhood form of HPP.

\section{Case 1: 7-year-old boy}

The patient began experiencing pain in his lower legs at 2 years of age. At 4 years, he had pain in the upper arms and exhibited premature loss of three deciduous teeth. The serum ALP level was 197 IU/L (reference value for this age: 430-1200 IU/L). X-ray findings of flaring at the distal ulnar metaphyseal end and cupping at the distal metaphyseal ends of both radii were noted. Genetic testing revealed that he had compound heterozygosity for c.613G >A (p.Ala205Thr) and c.1559delT (p.Leu520fs) of the $A L P L$ gene. We initiated AA treatment, and there was consequent improvement in his symptoms of pain in the lower extremities and a gradual decline in the rate of premature loss of deciduous teeth. The patient's height increased from $104 \mathrm{~cm}(-0.8 \mathrm{SD}$ below the mean for his age group as per the 2000 growth survey) [21] to $117 \mathrm{~cm}$ $(-0.5 \mathrm{SD}) 2$ years after the initial presentation. His $6 \mathrm{MWT}$ result improved from $350 \mathrm{~m}$ (70\% of normal) to $400 \mathrm{~m}$ (75\% of normal) after initiation of AA treatment. Both cupping and flaring at the forearm had disappeared 2 years after the commencement of AA treatment. He experienced mild lipoatrophy at injection sites. This case is that of a mild childhood form of HPP.

\section{Case 2: 12-year-old girl}

The patient had poor weight gain, and her radiographs showed rachitic changes at the ends of the bones of the trunk at 6 months of age. At 1 year of age, she was suspected to have HPP as her serum ALP levels were in the range of 260-380 IU/L (reference value for this age: 395$1289 \mathrm{IU} / \mathrm{L}$ ), but she had no other symptoms. Thereafter, her height was -1.0 to -2.5 SD below the mean for her age group as per the 2000 growth survey [21]. At 8 years of age, she experienced neck pain and lumbago. There were no abnormalities on imaging tests, and HPP was once again suspected. Genetic analysis revealed a heterozygous variant of the ALPL gene, c.984_986delCTT (p.Phe327del). Her father had the same variant but was asymptomatic; therefore, this variant was not thought to be the cause of her symptoms. The patient was rehabilitated and treated with anti-inflammatory drugs. At the age of 10 years, she became unable to attend school on account of her inability to walk, carry her bag by herself, or play with her friends because of pain in her extremities (Additional file 1). She was referred to our hospital to commence AA treatment as the serum PLP level was elevated at $164.4 \mathrm{nmol} / \mathrm{L}$ (reference value 14.5$57.3 \mathrm{nmol} / \mathrm{L}$ ); subsequently, the pain in her extremities gradually decreased. Approximately 3-4 months after the initiation of AA treatment, she was able to join an athletic festival without the support of any anti-inflammatory drugs. Six months after AA treatment initiation, she did not have any difficulty in attending school (Additional file 1). Her $6 \mathrm{MWT}$ result improved from $380 \mathrm{~m}$ (60\% of normal) to $433 \mathrm{~m}$ ( $68 \%$ of normal) after AA treatment initiation. She experienced mild lipoatrophy and mild redness at injection sites. This case is that of a mild childhood form of HPP.

\section{Case 3: 3-year-old boy}

The patient lost five deciduous teeth prematurely with roots intact when he was aged 1 year and 4 months and one tooth when aged 1 year and 5 months. He visited a dentist who suspected HPP. He was then referred to our hospital for further investigation and treatment. His height was $74 \mathrm{~cm}(-2.0 \mathrm{SD})$, and radiography showed bilateral distal ulna cupping. The serum ALP level was $79 \mathrm{IU} / \mathrm{L}$ (reference value for this age: 395-1339 IU/L), and the c.526G $>\mathrm{A}$ (p.Ala176Thr) and c.920C $>\mathrm{T}$ (p.Pro307Leu) $A L P L$ variants were detected. We started AA treatment at age 1 year and 5 months. He lost one tooth when he was aged 2 years and 3 months; since then, there had been no tooth loss till the last follow-up at age 3 years and 2 months. His height increased from $74 \mathrm{~cm}$ $(-2.0 \mathrm{SD})$ to $87 \mathrm{~cm}(-1.8 \mathrm{SD}) 1$ year and 7 months after AA treatment initiation. Radiography showed disappearance of cupping of the distal ulna 2 years after AA treatment initiation. He experienced mild lipoatrophy with mild redness at injection sites. This case is that of a mild childhood form of HPP.

\section{Case 4: 12-year-old boy}

At 9 years of age, the patient complained of pain in the right knee and hip joint and was referred to an orthopedic surgeon. No abnormalities were noted on radiography, but the patient was monitored. Over the next several 
months, he developed pain in his chest and extremities and was referred to our hospital for a detailed investigation. Blood examination revealed a low serum ALP level of $421 \mathrm{IU} / \mathrm{L}$ (reference value for this age: 470-1500 IU/L). Genetic testing for the ALPL gene exhibited a heterozygous c.1559delT variant (p.Leu520fs). Based on our experience with treating case 2 of this series, that of a patient with HPP with a heterozygous variant responsive to AA treatment, we likewise commenced AA treatment in case 4 at the age of 11 years. The patient's pain gradually subsided after the introduction of AA treatment. He experienced mild lipoatrophy with mild redness at injection sites. This case is that of a mild childhood form of HPP.

\section{Case 5: 12-year-old boy}

At the age of 7 years, the patient had a short stature at $-2.0 \mathrm{SD}$ as per the 2000 growth survey [21]. Blood examination by a general practitioner revealed that the serum ALP level was 338 IU/L (reference value for this age: 450-1250 IU/L). He was referred to our hospital at the age of 9 years for further examination and treatment. Orthopedic examination and radiography revealed a spur on the right radial diaphysis, lumbar kyphosis, strong pelvic anteversion, and hip flexion contracture. His serum ALP level continued to remain low, and he had developed bilateral ankle pain, which prompted us to perform further examinations. The $A L P L$ variants c.382G >A (p.Val128Met) and c.529G >A (p.Ala177Thr) were found, and AA treatment was initiated. His height increased from $127 \mathrm{~cm}(-1.9 \mathrm{SD})$ to $142 \mathrm{~cm}(-1.4 \mathrm{SD})$ in 2 years and 3 months, and his pain gradually improved after the introduction of AA treatment, although radiographic findings did not improve. He experienced lipohypertrophy and lipoatrophy with skin redness at injection sites. This case is that of a mild childhood form of HPP.

\section{Case 6: 18-year-old girl}

This patient, the older sister of patient 5 of this series, experienced left knee pain at the age of 10 years and was treated surgically after a diagnosis of meniscus injury, but the knee pain persisted. She was also diagnosed with scoliosis at 13 years of age. When she was 15 years old, her younger brother (case 5) was diagnosed with HPP. Consequently, she was suspected to have the same disease. Blood examination revealed a low serum ALP level of $140 \mathrm{IU} / \mathrm{L}$ (reference value for this age: 120-570 IU/L). We diagnosed her with HPP. Genetic testing showed the same $A L P L$ variants as her brother, which was consistent with the diagnosis. At 16 years of age, AA treatment was initiated. Her knee pain gradually improved, and she experienced considerably less fatigue in her daily life after the initiation of AA treatment. Her $6 \mathrm{MWT}$ result also improved from $356 \mathrm{~m}$ (54\% of normal) to $410 \mathrm{~m}$ (65\% of normal) after AA treatment initiation. She experienced mild lipoatrophy at injection sites. This case is that of a mild childhood form of HPP.

\section{Case 7: 5-year-old boy}

At 4 years of age, the patient lost a deciduous tooth with the root intact. He was taken to a general practitioner, and his serum ALP level was found to be $116 \mathrm{IU} / \mathrm{L}$ (reference value for this age: 430-1200 IU/L). Thereafter, he was referred to our hospital for further examination and treatment. Radiography revealed band sclerosis at the distal femoral metaphyseal end. A genetic test revealed $A L P L$ variants-c.572A $>$ G (p.Glu191Gly) and c.1559delT (p.Leu520fs) - and the diagnosis of HPP. Nine months after AA treatment initiation, he had not experienced any further premature loss of deciduous teeth. Radiographs showed that band sclerosis tended to improve, with no significant change in the bone permeability 3 months after AA treatment initiation. He experienced mild redness at injection sites. This case is that of a mild childhood form of HPP.

\section{Case 8: 21-year-old male}

At birth, the patient had a shorter right thigh and a shorter right upper arm than the left thigh and left upper arm, respectively. He had no significant complications other than the differences in the length of his upper arms and lower legs. At the age of 2 years, his serum ALP level was $103 \mathrm{IU} / \mathrm{L}$ (reference value of this age: 410-1250 IU/L). Subsequently, a short stature at approximately -2.0 SD as per the 2000 growth survey [21] and periodontal disease were noted. At the age of 8 years, he was referred to our hospital for surgical correction of the difference in the length of his lower legs, and the operation was performed. When the patient was 15 years old, AA was approved in Japan. He had bilateral kidney calcification at this time. Genetic testing was performed before treatment, and the $A L P L$ gene variants c.979T $>C$ (p.Phe327Leu) and c1559delT (p.Leu520fs) were found; hence, AA was commenced. Subsequently, his 6MWT results improved from $463 \mathrm{~m}$ (67\% of normal) to $560 \mathrm{~m}$ (81\% of normal), and kidney calcification also resolved. He experienced lipohypertrophy and lipoatrophy with skin redness at injection sites. This case is that of a benign prenatal form of HPP.

\section{Case 9: 9-year-old girl}

Fetal ultrasound at 29 weeks of age revealed bilateral femoral deformities. A low serum ALP level (96 IU/L; reference value for this age: 530-1610 IU/L) at birth had shown and she was diagnosed HPP. Genetic testing revealed $A L P L$ variants, namely c.568_570delAAC (p.Asn190del) and c.979T $>C \quad$ (p.Phe327Leu). She 
subsequently had a short stature of less than $-2.0 \mathrm{SD}$ and also developed bilateral kidney calcification. AA treatment was started at the age of 4 years for short stature, although treatment was discontinued within one week due to injection pain. When she was 6 years and 3 months old, AA treatment was resumed due to concerns regarding her short stature and kidney calcification. She experienced gradual alleviation in fatigue after the initiation of AA treatment, although mild kidney calcification persisted after 1 year and 4 months of AA treatment. Her height had increased from $103 \mathrm{~cm}(-2.4$ $\mathrm{SD})$ to $119 \mathrm{~cm}(-2.3 \mathrm{SD})$ at age 3 years and 1 month. She experienced lipohypertrophy and lipoatrophy with skin redness at injection sites. This case is that of a benign prenatal form of HPP.

\section{Case 10: 2-year-old girl}

The patient had prominently shorter limbs than normal since the fetal period and was born at 38 weeks and 3 days of gestation. She had severe respiratory failure immediately after birth and was therefore intubated. Blood test results after birth revealed a low serum ALP level of $8 \mathrm{IU} / \mathrm{L}$ (reference value for this age: 530-1610 IU/L). Consequently, she was clinically diagnosed as having HPP and was started on AA treatment and vitamin B6 supplements on day 0 . A genetic test later revealed the $A L P L$ variants c.1333T $>C$ (p.Ser445Pro) and c.1559delT (p.Leu520fs) and the diagnosis of HPP. The patient has sensorineural hearing loss that is gradually improving. She needs respiratory support during the night, but the respirator can be taken off during the daytime. She could pull herself up at the age of 2 years. Although she still had severe short stature, her height increased from $35 \mathrm{~cm}$ $(-6.5 \mathrm{SD})$ to $73 \mathrm{~cm}(-4.8 \mathrm{SD})$ in 2 years and 4 months after AA treatment initiation. She experienced mild lipoatrophy at injection sites. This case is that of a perinatal form of HPP.

To summarize, our case series reports the details of AA treatment experience in ten patients with HPP from 9 families from January 2015 to November 2019 (median [interquartile range] age 11.0 years [7.6-12.5] years; $60 \%$ male patients) (Table 2). There was one case of perinatal form, two cases of benign prenatal form, and seven cases of mild childhood form of HPP. The most common symptom at onset was pain in the limbs (three out of ten patients: cases 1,4 , and 6 ). The most common symptoms during the clinical course of the disease were pain (five out of ten patients: cases 1,2, 4, 5, and 6) and short stature (five out of ten patients: cases 2, 3, 8, 9, and 10). Two patients (cases 8 and 9) had bilateral kidney calcification. All ten patients had low serum ALP levels before AA treatment as compared with the age-matched reference range.
Genetic analysis showed that eight out of ten patients had compound heterozygosity, and two patients (cases 2 and 4) had only one heterozygous variant. Five out of ten patients had the c.1559delT (p.Leu520fs) variant, which is common in Japan [10]. In contrast, only two patients had the c.979T $>$ C (Phe310Leu) variant, which is also common in Japan [10].

All patients showed improvements in clinical symptoms after AA treatment, such as increase in height, pain alleviation, fatigue alleviation, improvement in premature deciduous tooth loss, better performance in the 6MWT, or improvement in respiratory insufficiency. All five patients (cases 1, 2, 4, 5, and 6) who experienced pain in extremities had alleviation of pain after AA treatment initiation. In one patient (case 3) with premature loss of deciduous teeth with the roots intact, the interval of losing teeth seems to have reduced after initiation of AA treatment. In case 7, the patient had not experienced further premature loss of deciduous teeth 9 months after AA treatment. Clinical improvements in patients with the benign prenatal form (cases 8 and 9) were increased distance in the $6 \mathrm{MWT}$, and fatigue alleviation. Kidney calcification resolved in case 8 , but not in case 9. The patient in case 10 had severe short stature, with a height of $73 \mathrm{~cm}$ $(-4.8 \mathrm{SD})$ at 2 years and 4 months of age even after the initiation of AA treatment (her height was $-6.5 \mathrm{SD}$ at birth), although respiratory insufficiency improved, and motor, mental, and auditory development progressed gradually. Biochemical analysis after the initiation of AA treatment showed a decrease in PLP, PLP/ $\mathrm{PL}$, and PEA values. These changes are consistent with the pathophysiology of the disease when treated with AA. Serum PLP levels of patients in cases 2, 6, and 9 decreased to subnormal levels after AA treatment initiation.

In three patients (cases 5, 6, and 9), AA treatment was either discontinued temporarily or the total dose was reduced owing to injection site reaction or fever. Patient 5 resumed adequate dose AA treatment 6 months after dose reduction. Patient 6 continues to receive AA treatment twice a week instead of three times a week owing to pain due to AA treatment injection. Patient 9 resumed AA treatment 1 year and 10 months after discontinuation due to concerns about her short stature and kidney calcification. We did not encounter anaphylactic shock, significant fluctuations of serum calcium and phosphorus levels, or ectopic calcification due to long-term treatment with AA as far as we know. All patients experienced injection site reaction to varying degrees and some patients experienced lipohypertrophy and lipoatrophy (Additional file 2). There were no other complications that could be caused by AA. 


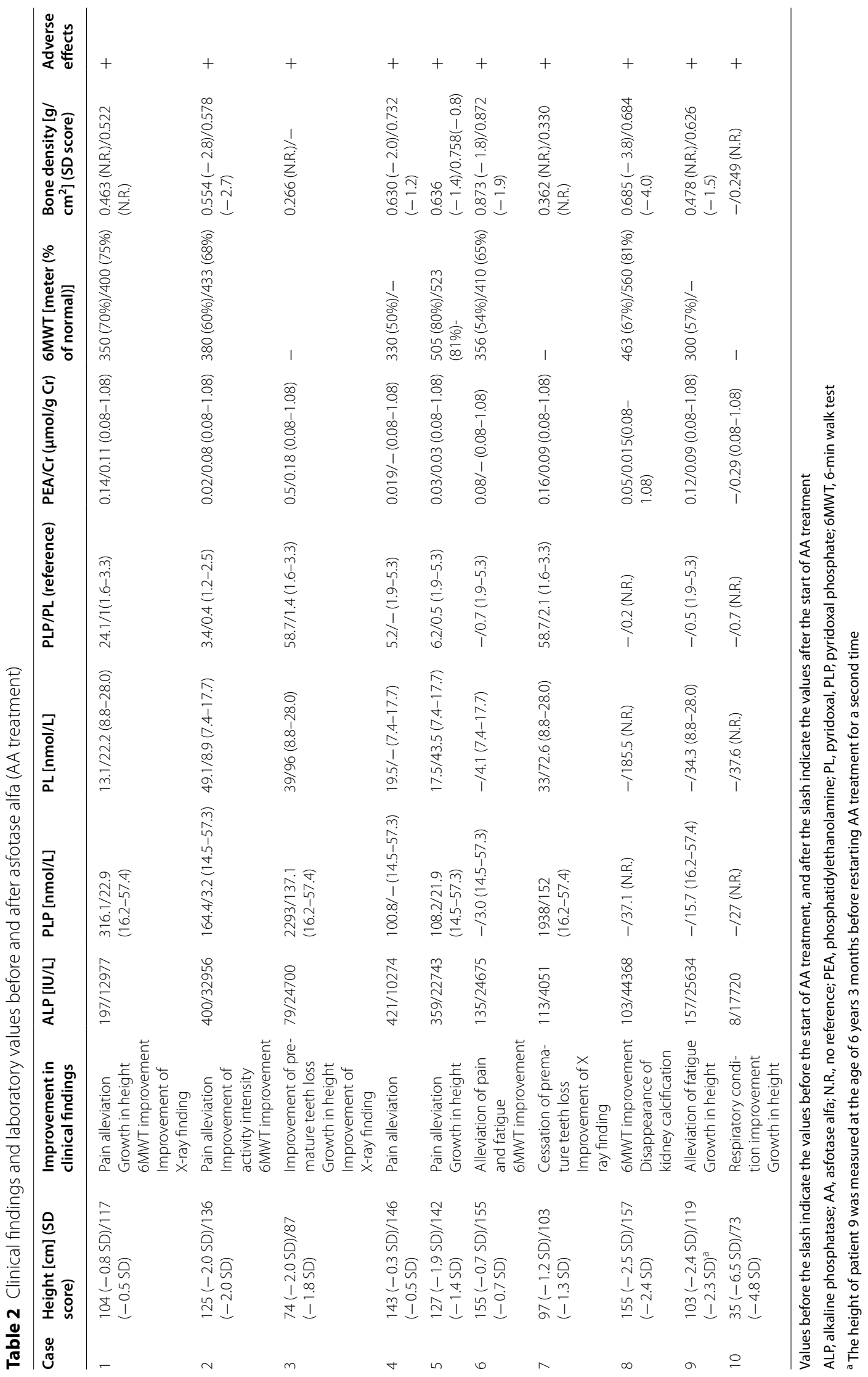




\section{Discussion}

This was a single-center cohort study in Japan describing the clinical course of patients with HPP treated using AA. We have shown some efficacy of AA treatment in pediatric-onset HPP without serious complications; however, there were some limitations in our study. First, effectiveness of AA in reducing pain and fatigue was assessed based on the subjective responses of the patients or their parents. Second, our study group was a single-center cohort. Nevertheless, we believed that presenting the clinical courses of patients with HPP during AA treatment would benefit clinicians treating patients with HPP.

Although the efficacy of AA in the perinatal and infantile form of HPP has been demonstrated in several countries, including Japan, the number of patients with the childhood and adult forms of mild HPP was comparatively lower [15, 22]. There is a monitoring guidance for patients with HPP treated with AA, which suggests regular monitoring of biochemistry, skeletal radiographs, respiratory function, growth, pain, mobility and motor function, and quality of life [23].In our study, not all patients had rachitic changes on radiographs, and thus these patients with the childhood form appeared to have milder findings compared to those in the clinical trial [22], wherein all patients had rachitic changes on radiographs and the childhood form was severe. In our study, clinical findings such as short height, premature loss of teeth, poor performance in the $6 \mathrm{MWT}$, or respiratory insufficiency were improved after initiating AA treatment. Some patients reported improvements regarding only subjective parameters such as pain and fatigue. To quantify these symptoms, it is necessary to use dedicated questionnaires. Although there is no published report about AA treatment reducing premature loss of teeth in HPP, it seems that AA treatment reduced the interval of losing teeth in some of our patients. It has been demonstrated that DXA scans are likely not useful in HPP, though we saw improvement in bone density with DXA scans in some patients [24].

Long-term treatment of patients with mild HPP with a conventional dosage of AA might have a hypothetical risk of leading to excessively low inorganic pyrophosphate levels, which may have effects such as ectopic mineralization. A similar condition is generalized arterial calcification of infancy (GACI), which is characterized by reduced levels of PPi in plasma and can be lethal in infancy. In GACI, it has been postulated that reduced PPi is a major determinant for ectopic mineralization, since PPi is a powerful mineralization inhibitor [25]. In Japan, AA dosage can be adjustable at the discretion of the attending physician, although there is no guideline or evidence about dosage adjustment. In cases 2,6 , and 9, we considered reducing the dosage of AA since their serum PLP level dropped to a subnormal level.
Case 10 with perinatal HPP continued to have severe short stature even though the growth rate improved considerably after the initiation of AA treatment (from -6.5 to $-4.8 \mathrm{SD}$ ). We could not draw a comparison between the clinical signs before and after the initiation of AA treatment with respect to case 10 as AA treatment was initiated immediately after birth. The natural prognosis of perinatal HPP is poor, with death generally occurring within 1 year [26]. Therefore, we can say that AA treatment improved the patient's prognosis.

Although genetic analysis is recommended for diagnosing HPP, a detailed patient history and clinical examination are the crucial first steps. There might be patients with heterozygous $A L P L$ variants, like in case 2, who respond to AA treatment. We should comprehensively consider the indications for starting AA treatment in patients with HPP and closely monitor their clinical improvement while evaluating the safety and complication of long-term AA treatment for each patient. Further studies are needed to explore the improvements in clinical signs and to assess the safety and complications associated with various forms of HPP after AA treatment.

\section{Conclusions}

We reported the single-center cohort in Japan describing the clinical course of patients with HPP, treated with AA. Every patient seemed to benefit from the AA treatment without serious adverse effects. We will continue to conduct future studies with long-term follow-up periods to investigate the efficacy and safety of AA treatment in patients with HPP.

\section{Abbreviations}

AA: Asfotase alfa; DXA: Dual X-ray absorption; HPP: Hypophosphatasia; PEA: Phosphoethanolamine; PLP: Pyridoxal 5' phosphate; SD: Standard deviation; TNSALP: Tissue-nonspecific alkaline phosphatase; 6MWT: 6-Minute walk test.

\section{Supplementary Information}

The online version contains supplementary material available at https://doi. org/10.1186/s13023-022-02230-y.

Additional file 1. Video record of patient 2 demonstrating the patient's inability to walk and the subsequent improvement in pain and walking ability after asfotase alfa (AA) initiation. Description of data: At the age of 10 years, the patient could not attend school on account of her inability to walk, carry her bag by herself, or play with her friends because of pain. The pain in her extremities gradually improved after the introduction of AA treatment. Three to four months after AA treatment initiation, she could join an athletic festival without the support of any anti-inflammatory drugs. Six months after AA treatment initiation, she did not have any difficulty attending school.

Additional file 2. Skin changes noted in each case.

Additional file 3. Japanese prevalence of the each variant with Japanese Multi Omics Reference Panel. 


\section{Acknowledgements}

We would like to thank Editage (www.editage.com) for English language editing.

\section{Authors' contributions}

YS, TW, and MT drafted the article. MS, TM, TE, TF, AM, KI, and TT critically reviewed the manuscript. TA measured pyridoxal 5'-phosphate and pyridoxal, and critically reviewed the manuscript. KM is the acting guarantor. All authors have read and approved the final manuscript.

\section{Funding}

No funding was received for this study.

\section{Availability of data and materials}

The datasets used and/or analyzed during the current study are available from the corresponding author on reasonable request.

\section{Declarations}

\section{Ethics approval and consent to participate}

This is an observational retrospective patient report that did not involve any research-based patient intervention. All diagnostic procedures and treatment protocols were based on established protocols intended to diagnose and treat the patients. No aspect of the case series is in contradiction with the Helsinki Declaration of 1975, as revised in 2000. The assay of pyridoxal phosphate and pyridoxal was approved by the Research Ethics Board at Okayama University Hospital (Approval No. 1603-012). Written informed consent for publication of the data and for performing measurements of PLP and PL were obtained from the patients or patient's parents (in the cases of patients below 18 years of age).

\section{Consent for publication}

Written informed consent for publication of the data, measurements of PLP and $\mathrm{PL}$, and the video record of patient 2 was obtained from the patient's parents.

\section{Competing interests}

The authors declare no competing interests.

\section{Author details}

${ }^{1}$ Center for Medical Genetics, Chiba Children's Hospital, 579-1 Heta-cho, Midori-ku, Chiba City, Chiba Prefecture 266-0007, Japan. ${ }^{2}$ Department of Metabolism, Chiba Children's Hospital, Chiba, Japan. ${ }^{3}$ Department of Neonatology, Chiba Children's Hospital, Chiba, Japan. ${ }^{4}$ Department of Child Neurology, Okayama University Hospital, 2-5-1 Shikata-cho, Kita-ku, Okayama city, Okayama Prefecture 700-8558, Japan.

Received: 14 July 2021 Accepted: 6 February 2022

Published online: 23 February 2022

\section{References}

1. Mornet E. Hypophosphatasia. Orphanet J Rare Dis. 2007;2:40. https://doi. org/10.1186/1750-1172-2-40

2. Weiss MJ, Ray K, Henthorn PS, Lamb B, Kadesch T, Harris H. Structure of the human liver/bone/kidney alkaline phosphatase gene. J Biol Chem. 1988;263:12002-10.

3. Seefried L, Kishnani P, Moseley S, Denker AE, Watsky E, Whyte MP, et al. Pharmacodynamics of asfotase alfa in adults with pediatric-onset hypophosphatasia. Bone. 2021;142:115664. https://doi.org/10.1016/j. bone.2020.115664.

4. Millan JL, Whyte MP. Alkaline phosphatase and hypophosphatasia. Calcif Tissue Int. 2016;98:398-416. https://doi.org/10.1007/s00223-015-0079-1.

5. Addison WN, Azari F, Sørensen ES, Kaartinen MT, McKee MD. Pyrophosphate inhibits mineralization of osteoblast cultures by binding to mineral, up-regulating osteopontin, and inhibiting alkaline phosphatase activity. J Biol Chem. 2017;282:15872-83. https://doi.org/10.1074/jbc.M701116200.

6. Anderson HC. Matrix vesicles and calcification. Curr Rheumatol Rep. 2003;5:222-6. https://doi.org/10.1007/s11926-003-0071-z.
7. Fleisch H, Bisaz S. Mechanism of calcification: inhibitory role of pyrophosphate. Nature. 1962;195:911. https://doi.org/10.1038/195911a0.

8. Lin EL, Gottesman GS, McAlister WH, Bijanki VN, Mack KE, Griffin DM, et al. Healing of vitamin D deficiency rickets complicating hypophosphatasia suggests a role beyond circulating mineral sufficiency for vitamin $D$ in musculoskeletal health. Bone. 2020;136:115322. https://doi.org/10.1016/j. bone.2020.115322.

9. Wenkert D, McAlister WH, Coburn SP, Zerega JA, Ryan LM, Ericson KL, et al. Hypophosphatasia: nonlethal disease despite skeletal presentation in utero (17 new cases and literature review). J Bone Miner Res. 2011;26:2389-98. https://doi.org/10.1002/jbmr.454.

10. Michigami T, Uchihashi T, Suzuki A, Tachikawa K, Nakajima S, Ozono K. Common mutations F310L and T1559del in the tissue-nonspecific alkaline phosphatase gene are related to distinct phenotypes in Japanese patients with hypophosphatasia. Eur J Pediatr. 2005;164:277-82. https:// doi.org/10.1007/s00431-004-1612-9.

11. Michigami T, Ohata Y, Fujiwara M, Mochizuki H, Adachi M, Kitaoka T, et al. Clinical practice guidelines for hypophosphatasia. Clin Pediatr Endocrinol. 2020;29:9-24. https://doi.org/10.1297/cpe.29.9.

12. Daniel AB, Saraff V, Shaw NJ, Yates R, Mughal MZ, Padidela R. Healthcare resource utilization in the management of hypophosphatasia in three patients displaying a spectrum of manifestations. Orphanet J Rare Dis. 2018;13:142. https://doi.org/10.1186/s13023-018-0869-4.

13. Whyte MP. Hypophosphatasia - aetiology, nosology, pathogenesis, diagnosis and treatment. Nat Rev Endocrinol. 2016;12:233-46. https://doi.org/ 10.1038/nrendo.2016.14.

14. Mornet E, Taillandier A, Domingues C, Dugour SA, Benaloun E, Lavaud $\mathrm{N}$, et al. Hypophosphatasia: a genetic-based nosology and new insights in genotype-phenotype correlation. Eur J Hum Genet. 2021;29:289-99. https://doi.org/10.1038/s41431-020-00732-6.

15. Kitaoka T, Tajima T, Nagasaki K, Kikuchi T, Yamamoto K, Michigami T, et al. Safety and efficacy of treatment with asfotase alfa in patients with hypophosphatasia: results from a Japanese clinical trial. Clin Endocrinol. 2017:87:10-9. https://doi.org/10.1111/cen.13343.

16. Whyte MP, Madson KL, Phillips D, Reeves AL, McAlister WH, Yakimoski A, et al. Asfotase alfa therapy for children with hypophosphatasia. JCl Insight. 2016;16(1):e85971. https://doi.org/10.1172/jci.insight.85971.

17. Tanaka T, Yamashita A, Ichihara K. Reference intervals of clinical tests in children determined by a latent reference value extraction method (in Japanese). J J.pn Pediatr Soc. 2008;112:1117-32.

18. Whyte MP, Zhang F, Wenkert D, McAlister WH, Mack KE, Benigno MC, Coburn SP, Wagy S, Griffin DM, Ericson KL, Mumm S. Hypophosphatasia: validation and expansion of the clinical nosology for children from 25 years experience with 173 pediatric patients. Bone. 2015;75:229-39. https://doi.org/10.1016/j.bone.2015.02.022.

19. Akiyama T, Hayashi Y, Hanaoka Y, Shibata T, Akiyama M, Tsuchiya H, et al. Pyridoxal 5'-phosphate, pyridoxal, and 4-pyridoxic acid in the paired serum and cerebrospinal fluid of children. Clin Chim Acta. 2017:472:11822. https://doi.org/10.1016/j.cca.2017.07.032.

20. Japanese Ministry of Health, Labour and Welfare. National growth survey on preschool children, Tokyo, Japan (in Japanese). 2000. https://www. mhlw.go.jp/houdou/0110/h1024-4.html. Accessed on 25 June 2021.

21. Japanese Ministry of Education, Culture, Sports, Science and Technology. Report of the national growth survey of normal Japanese children, 5-17 years of age, Tokyo, Japan (in Japanese). 2003. https://www.e-stat.go.jp/ stat-search/files? page $=1 \&$ layout $=$ datalist\&touke $=00400002 \&$ tstat $=$ $000001011648 \&$ cycle $=0 \&$ tclass $1=000001022251 \&$ tclass $2=0000010222$ 52\&stat_infid $=000001199546 \&$ tclass3val=0. Accessed on 25 June 2021.

22. Whyte MP, Greenberg CR, Salman NJ, Bober MB, McAlister WH, Wenkert $D_{\text {, }}$ et al. Enzyme-replacement therapy in life-threatening hypophosphatasia. N Engl J Med. 2012;366:904-13. https://doi.org/10.1056/NEJMoa1 106173.

23. Kishnani PS, Rush ET, Arundel P, Bishop N, Dahir K, Fraser W, et al. Monitoring guidance for patients with hypophosphatasia treated with asfotase alfa. Mol Genet Metab. 2017;122:4-17. https://doi.org/10.1016/j.ymgme. 2017.07.010.

24. Simmons JH, Rush ET, Petryk A, Zhou S, Martos-Moreno GÁ. Dual X-ray absorptiometry has limited utility in detecting bone pathology in children with hypophosphatasia: a pooled post hoc analysis of asfotase alfa clinical trial data. Bone. 2020;137:115413. https://doi.org/10.1016/j.bone. 2020.115413. 
25. Veiga-Lopez A, Sethuraman V, Navasiolava N, Makela B, Olomu I, Long $R$, et al. Plasma inorganic pyrophosphate deficiency links multiparity to cardiovascular disease risk. Front Cell Dev Biol. 2020;8:573727. https://doi. org/10.3389/fcell.2020.573727.

26. Leung EC, Mhanni AA, Reed M, Whyte MP, Landy H, Greenberg CR.

Outcome of perinatal hypophosphatasia in manitoba mennonites: a retrospective cohort analysis. JIMD Rep. 2013;11:73-8. https://doi.org/10. 1007/8904_2013_224.

\section{Publisher's Note}

Springer Nature remains neutral with regard to jurisdictional claims in published maps and institutional affiliations.

- fast, convenient online submission

- thorough peer review by experienced researchers in your field

- rapid publication on acceptance

- support for research data, including large and complex data types

- gold Open Access which fosters wider collaboration and increased citations

- maximum visibility for your research: over $100 \mathrm{M}$ website views per year

At BMC, research is always in progress.

Learn more biomedcentral.com/submissions 